\title{
TTR
}

Traduction, terminologie, re?daction

\section{La trahison créatrice : anatomie du transfert notionnel dans les cultures asiatiques}

\section{Alain Rocher}

Volume 6, numéro 2, 2e semestre 1993

Traduction, mixité, politique

URI : https://id.erudit.org/iderudit/037149ar

DOI : https://doi.org/10.7202/037149ar

Aller au sommaire du numéro

\section{Éditeur(s)}

Association canadienne de traductologie

ISSN

0835-8443 (imprimé)

1708-2188 (numérique)

Découvrir la revue

\section{Citer cet article}

Rocher, A. (1993). La trahison créatrice : anatomie du transfert notionnel dans les cultures asiatiques. TTR, 6(2), 11-37. https://doi.org/10.7202/037149ar

\section{Résumé de l'article}

La trahison créatrice : anatomie du transfert notionnel dans les cultures asiatiques - Nous proposons une lecture constructiviste du phénomène de transfert conceptuel dans les traditions philosophiques et religieuses de la Chine et du Japon. Par transfert, nous entendons aussi bien l'emprunt d'une notion isolée et son insertion dans un nouveau système culturel que la translation d'un ensemble catégoriel complexe. Nous nous intéressons surtout aux distorsions sémantiques qui se produisent lors du transfert (et à ces cas extrêmes que sont les transformations délibérées) pour illustrer l'hypothèse suivante : l'importation d'un concept est toujours un acte de création. Nous soulignons l'importance des structures intentionnelles qui marquent le geste de translation : prospection, pré-interprétation, stratégies d'utilisation, etc. Cette hypothèse nous permet d'étudier la traduction philosophique en faisant l'économie de la problématique de la vérité, et ensuite de contester l'étiquette de " cultures de la copie » trop souvent apposée aux traditions d'Extrême-Asie.
Tous droits réservés @ C TTR: traduction, terminologie, rédaction — Les auteurs, 1993
Ce document est protégé par la loi sur le droit d'auteur. L'utilisation des services d'Érudit (y compris la reproduction) est assujettie à sa politique d'utilisation que vous pouvez consulter en ligne.

https://apropos.erudit.org/fr/usagers/politique-dutilisation/ 


\section{La trahison créatrice: anatomie du transfert notionnel dans les cultures asiatiques ${ }^{1}$}

\section{Alain Rocher}

«Vouloir traduire l'esprit est une ambition si démesurée et si fantomatique qu'on peut bien la considérer comme inoffensive. Vouloir traduire la lettre demande une précision si extravagante qu'il n'y a pas de risque qu'on l'entreprenne."

J. L. Borges, "les Traducteurs des Mille et une nuits», O.C., vol. I, Pléiade, p. 420.

«Son infidélité, son heureuse infidélité créatrice, voilà ce qui doit nous importer.»

J. L. Borges, ibid., p. 432.

\section{Introduction}

On connaît les affres d'Ibn Ruchd, qui butait sur l'intraduisibilité de la notion de «tragœdia». Après avoir cherché en vain une

1. Ce travail a été facilité par une subvention triennale du Programme d'aide aux nouveaux chercheurs du Québec (FCAR 1992-1995). Je tiens à remercier ici $M^{\text {lle }}$ S. Boyer, dont les compétences, tant dans le domaine du bouddhisme que dans celui de l'informatique, m'ont été extrêmement précieuses au stade de la recherche bibliographique dans les bibliothèques américaines et canadiennes. M. G. Tardif a bien voulu collaborer à mon projet de recherche en qualité d'assistant: qu'il trouve ici l'expression de ma gratitude. 
correspondance arabe, l'auteur du «Tahafut at Tahafut ${ }^{2}$ » définissait implicitement le transfert catégoriel comme l'art du factice, en montrant que l'unique recours laissé au maître des différences était d'inventer l'équivalence introuvable. Car, malgré les apparences, l'anecdote raconte autre chose que l'histoire d'un échec. Derrière le geste de clôture qui consiste à réduire l'inconnu au connu (réaction prévisible chez un penseur qui affirme que tout a déjà été dit) se cache ce que nous appellerons l'impératif de transformation. La faute d'Ibn Ruchd réside moins dans le fameux contresens (tragédie = panégyrique) que dans le choix $d^{\prime}$ un transfert infécond. Lorsqu'elle est assumée et transplantée en terrain fertile, l'inexactitude révèle tout son pouvoir créateur. Plus que le traducteur d'Aristote, le cas de Dôgen ${ }^{3}$ offre une riche matière à la réflexion en ce qu'il place magistralement le transfert conceptuel au-delà de la problématique de l'erreur. Même lorsqu'il traduit, le philosophe se doit d'être un inventeur de concepts. Transformant délibérément la thèse centrale du Mahâparinirvâna Sûtra ${ }^{4}$ selon laquelle tous les êtres possèdent la nature du bouddha parfait, le philosophe japonais, envers et contre la philologie, donne une nouvelle lecture (tout l'étant est la nature du bouddha), qui inscrit le message du Sûtra dans le

2. Le "Tahafut at Tahafut» (La destruction de la destruction) d'Ibn Ruchd (1126-1198) est une réponse à l'anti-aristotélisme de l'ouvrage polémique d'Al Chazali (1058-1111) «Tahafut al Falasifat" (La destruction des philosophes).

3. Dôgen (1220-1253): c'est l'introducteur de la secte zen «Soto" au Japon. La tendance rigoriste de ce courant est illustrée par le passage célèbre du "Bendôwa»: "Vous n'avez besoin $\mathrm{ni}$ d'encens ni de prières, ni d'invocation du nom du Bouddha, ni de confession, ni d'écritures saintes. Asseyez-vous et méditez!» Sur le plan théorique, on lui doit une réflexion originale sur la notion d' «être-temps" («Shôbôgenzô», chap. Uji).

4. Mahâparanirvana Sûtra: en pâli Mahâparinibbâna Sutta. Ce sûtra décrit les derniers jours et la mort du Bouddha. Il inclut donc les dernières instructions du maître à ses disciples. 
phénoménalisme $e^{5}$ fondamental de la pensée japonaise. Ce caslimite sera notre fil $d^{\prime}$ Ariane dans notre essai d'analyse du mécanisme du «transfert conceptuel». Par ce terme, nous désignerons aussi bien l'emprunt d'une notion isolée et son insertion dans un nouveau système culturel, que la translation d'un ensemble catégoriel complexe.

Nous limiterons notre champ aux traditions philosophiques de la Chine et du Japon. Le choix du langage philosophique (ou religieux) s'impose tout naturellement au traductologue car la charge affective et conceptuelle des mots-clés du bouddhisme, du confucianisme ou du daoïsme entraine un phénomène de réification que le tranfert ne peut que favoriser. Cette tendance est souvent renforcée par les stratégies pédagogiques utilisées par les maîtres du savoir: l'on se souvient par exemple qu'An Shigao ${ }^{6}$ insistait dans son enseignement sur la présentation terme à terme des catégories du dhyâna.

Nous nous intéresserons tout particulièrement aux distorsions sémantiques qui se produisent lors du transfert (et à ces cas extrêmes que sont les transformations délibérées) pour illustrer notre hypothèse: l'importation d'un concept est toujours un acte de création. Il s'agira pour nous de souligner l'importance des structures intentionnelles qui accompagnent le geste de translation: prospection, pré-interprétation, stratégies d'utilisation, anticipation des spécificités de l'horizon de réception, etc. Cette hypothèse nous permettra d'abord d'étudier la traduction philosophique en faisant l'économie de la problématique de la vérité, et ensuite de contester l'étiquette de "cultures de la copie» trop souvent apposée aux traditions de $l^{\prime}$ Extrême-Asie.

5. Nous empruntons cette expression à Nakamura Hajime (cf. "Ways of thinking of Eastern People»).

6. An Shigao: d'origine parthe, ce moine bouddhiste fut l'un des premiers introducteurs du bouddhisme en Chine (et l'un des premiers traducteurs de l'organon bouddhiste). 
Nous appuierons notre analyse sur une combinaison méthodologique qui pourra sembler contre nature: la déconstruction et la théorie des systèmes. Non que nous cédions au plaisir dérisoire d'appliquer à un contexte exotique un appareil théorique en voie d'obsolescence, mais à seule fin de montrer que certaines des techniques derridiennes retrouvent spontanément les stratégies universelles de démantèlement conceptuel qui accompagnent les importations de systèmes philosophiques ou leurs transformations au sein du même horizon culturel. Notre deuxième outil s'inspirera du constructivisme pratiqué par le dernier Luhmann (assoupli par la lecture de Maturana) et nous montrerons comment les variantes «nationales» des grands systèmes de pensée asiatiques se constituent dans un rapport $d^{\prime}$ 'assimilation antagoniste aux cultures centrales.

\section{Typologie du transfert}

La nature même de notre hypothèse nous imposera un certain parti-pris dans le choix des exemples: nous nous intéresserons aux phénomènes de syncrétisme ou aux pratiques de translation conceptuelle qui ne problématisent pas l'authenticité philologique. Dans le bouddhisme chinois, par exemple, ce sont les tendances anciennes, antérieures à la fondation du Xiaoyao yuan ${ }^{7}$ et à la méthode du yanyishibian de l'équipe Dao'anKumarajiva, qui nous retiendront $t^{8}$. Loin d'être une façon

7. Xiaoyao yuan: la fondation de ce centre de traduction en $\mathbf{3 8 3}$ (placé sous le patronage du souverain) marque le point de départ officiel de la nouvelle école de traduction, préoccupée de rigueur philologique.

8. À l'époque de Dao'an, le travail de traduction est subdivisé en six fonctions (assumées par des spécialistes différents: koushou, bishou, chuanyan, koushou, zhengyi, jiaodui) qui, après Xuanzhuang se complexifieront encore (yizhu, zhengyi, zhengwen, duyu, bishou, zhuiwen, canyi, kanding, runwen, fanbei). Cette démultiplication des différents moments de la traduction dénote bien sûr un souci de rigueur philologique, comme l'indique la position stratégique du zhengyi, du 
d'adapter notre corpus à notre hypothèse, ce choix repose sur la conviction qu'en Asie les tendances syncrétiques ont exercé à long terme une influence plus profonde sur la construction des ensembles philosophiques ou religieux que la rigueur philologique des professionnels de la "nouvelle traduction».

\section{L'originaire introuvable}

Quand on aborde les traditions philosophiques chinoises, et, a fortiori, japonaises, aussi loin qu'on remonte dans le temps, l'on est toujours confronté à l'évidence d'un hétérogène déjà là. Il serait naïf de prétendre reconstruire une Weltanschauung originaire antérieure à toute importation. Dans l'histoire des idées, l'autochtonie ne peut être pensée que sur le mode du mythe. Il appartient à l'idéologie du néo-traditionalisme (fugu ou Kokugaku) ${ }^{9}$ d'asseoir sa légitimité sur cette illusion. Car

zhengwen, du canyi et du jiaodui. Notons que, même chez les techniciens de la nouvelle école, apparaît une conscience claire de l'inévitabilité des déformations sémantiques: Dao'an nomme "5 pertes» les déperditions de sens causées par le passage du sanskrit au chinois, et il va même jusqu'à établir une typologie des difficultés de traduction (les «san bu yi»). Le principe du "yanyi shibian" désigne la distinction des mots et des sens. Dans un même ordre d'idées, rappelons que la permanence de la polémique "wen pai/zhi pai» (école de la traduction fleurie/école de la traduction substantielle) montre indirectement que la solution technicienne des moines philologues ne pouvait résoudre tous les problèmes posés par le transfert.

9. Le néo-traditionalisme est une tendance qui prône le retour à la simplicité du style archaïque et à l'authenticité des valeurs de l'Antiquité. En Chine, ce mouvement prend souvent la forme d'une réaction contre le bouddhisme: ainsi Han Yu (768-824) se donne-t-il pour mission de restaurer le Tao. Au Japon, le Kokugaku (Études Nationales) se présente comme une réaction contre le néo-confucianisme et comme une redécouverte des racines prétendument vernaculaires de la culture nippone. $\mathrm{Ce}$ mouvement se développe au Japon entre le XVII et le XIX siècle. 
précisément, ce qui fonde la culture et la rend possible, ce n'est pas le développement spontané d'une Volksseele qui se reproduirait par parthénogénèse ou scissiparité, mais le jeu des variantes discursives et des systèmes qui se développent par allélophagic. Une culture ne peut se saisir comme telle que parce qu'elle trouve dans son répertoire notionnel des séries de différences qui lui donnent la possibilité de se paraphraser et de se transcoder.

Il est banal de mentionner que le daoïsme ancien repose sur une rélaboration de courants hétérogènes (théorie du yin et du yang, théorie des wu xing ${ }^{10}$, traditions mythologiques, etc.), tendance qui culminera dans le grand effort de synthèse de la dynastie des Han, et qui trouve dans l'œuvre de Huai Nan Wang $^{11}$ son exemple le plus caractéristique. Au Japon, l'on sait que le shintoïsme, loin de dériver directement du chamanisme pré-bouddhique, se constitue et se définit par rapport au bouddhisme en un travail d'assimilation antagoniste au terme duquel il se donne une architecture spécifique, un clergé hiérarchisé, restructure son panthéon et ses grands rituels.

Bref, l'originaire est introuvable: il ne peut exister que comme fiction ou comme geste thétique d'autocréation. C'est bien là le rôle de cette Annale ambiguë qu'est le Nihonshoki ${ }^{12}$. A la

10. Wu xing se traduit très approximativement par «cinq éléments" (le «xing" chinois ayant une valeur plus dynamique que le mot «élément»).

11. Auteur de la dernière grande synthèse taoïste, le «Huai Nan $\mathrm{Zi}$ ».

12. Kojiki (712) et Nihonshoki (720): Recueil de mythes, de généalogies impériales et de poèmes, le Kojiki raconte l'origine du pays et légitime la suprématie du clan impérial sur les autres clans. Le texte est rédigé à l'aide d'un système graphique mixte, mêlant les caractères idéographiques aux caractères phonétiques: il ne peut donc être lu à la chinoise. Le Nihonshoki est une annale beaucoup plus étendue que son aîné, construite sur le modèle des annales continentales et écrite en pur chinois. 
différence du Kojiki, le texte en kambun appréhende l'identité nationale dans son rapport à l'Autre. Le titre de l'ouvrage révèle le fondement imaginaire de cette dialectique de la reconnaissance. Le «Nihon» du composé «Nihonshoki» n'est ni un terme vernaculaire qui témoignerait $d^{\prime} u n e$ conscience de soi non encore distanciée (comme le mot incantatoire TöyöasiFaranötiakinönagaiFoakinömiduFo-nökuni ${ }^{13}$ du Kojiki), ni évidemment le terme dépréciatif utilisé dans les Annales $\operatorname{chinoises}^{14}$ (Wa no Kuni = pays des nains), mais un nom à la fois mythique et pseudo-savant, un euphémisme exprimé dans le langage du Grand Sujet civilisateur qu'était la Chine. L'expression «Source du soleil» nie l'excentricité géographique et culturelle du «dong yi» (barbares de l'est) qui dans les Annales désigne globalement Coréens, Japonais et les autres minorités d'Asie du Nord-Est. Par ce baptême ambigu, les compilateurs saisissent leur culture sous le regard d'autrui, mais, dans le même temps, ils cherchent à nier sa subordination. En se donnant un nom imaginaire, le Japon se constitue comme sujet culturel. Seule la mythologie savante (stade théologico-politique du shintoïsme) pourra prêter un langage à cette quête d'une identité nationale. L'on saisit alors l'importance du motif du "pays divin». Le passage de la chronique d'Ojin ${ }^{15}$ où l'on entend un étranger, le roi de Silla ${ }^{16}$, décrire le Japon comme un «kami no kuni», joue le rôle d'un acte de "recon-naissance», et illustre à merveille le caractère à la fois mythique et projectif de cette dialectique de la conscience. «À l'est se trouve un pays divin. On l'appelle Source

13. Terme magique qui peut se traduire par «La contrée fertile du champ de roseaux, pays aux mille et mille moissons".

14. Ces Annales sont le Hou Han Shu, le San Guo Zhi (livre Wei Shu) et le Sui Shu pour la période japonaise archaïque.

15. L'épisode est raconté dans le Nihonshoki, au chapitre du règne de l'empereur Ojin (cf. la traduction anglaise «Nihongi», Aston, 1896, 1972).

16. Silla est l'un des trois royaumes coréens de la période protohistorique. Les deux autres sont le Kokuryo (au nord) et le Paekche (à l'ouest). 
du Soleil (Nihon). Dans ce pays règne un souverain sacré. On l'appelle «empereur» (tennô/ sumera mikoto).

\section{Le transfert analogique}

Les concepts ou les systèmes de pensée «étrangers» sont rarement perçus comme une altérité menaçante au moment de leur importation. L'attitude la plus fréquente semble être de les assimiler au connu, comme si l'on ne s'intéressait d'abord qu'aux différences les plus digestes. Une autre raison, plus profonde, explique aussi le travail de l'homogénéisation: l'assimilation est déjà présente, a priori, dans l'acte même de représentation de l'autre et dans les choix qui orientent le geste de l'importation. On sait par exemple que, pendant les périodes Asuka et Nara, les Japonais décrivaient le bouddhisme comme une sorte de shintoïsme, alors même que Shotoku ${ }^{17}$ en avait montré, en acte, la radicale nouveauté. Rejet et polémiques ne surviennent que lorsque la nouvelle religion devient un enjeu politique dans les rivalités inter-claniques qui accompagnent la formation de l'État. Mais, même à ce stade, le fait de nommer le bouddha «tonariguni no kami» (kami du pays voisin) montre assez que le transfert est d'abord placé sous le signe du Même.

Dans l'histoire du bouddhisme chinois, c'est la technique du «geyi ${ }^{18}$ » qui représente le degré extrême de cette tendance

17. Sous le règne de l'impératrice Suiko (592-628), le prince héritier Shotoku Taishi exerce la réalité du pouvoir. Sa régence est marquée par d'importantes réformes qui toutes convergent vers le même but: la constitution d'un Etat unifié centré sur la personne de l'empereur. Il introduit un système de rang de cour fondé sur le mérite, promulgue une "constitution", adopte le calendrier chinois, favorise l'expansion du bouddhisme.

18. Le "geyi» fut utilisé jusqu'à la dynastie des Jin occidentaux (exclusivement). La mention du terme et la définition de la technique du geyi apparaissent pour la première fois dans le Gaoseng zhuan du bonze Huijiao (des Liang): «[...] en ce tempslà, tous les disciples de Ya étaient versés dans la littérature séculière, mais ne maîtrisaient pas les raisonnements 
assimilatrice. Pratique de traduction par vernacularisation, le geyi consiste à rendre les concepts du bouddhisme par des notions (et des idéogrammes) qui ont déjà une histoire et un sens spécifique dans les systèmes de pensée chinois, daoïsme ou confucianisme. Pour exprimer cela à la chinoise, le système notionnel des nei shu (livres intérieurs = sûtras) se trouve traduit dans le lexique offert par les wai shu (livres extérieurs = textes non bouddhiques), et donc interprété dans la perspective du xuan xue ${ }^{19}$. C'est. ainsi que le dharma sera assimilé au dao, ou encore les mahâbhûta traduits par wu xing (cinq éléments). Le sogdien Kang Senghui, lui, empruntera à la fois à Chuang zi et au confucianisme pour présenter les catégories bouddhistes aux Chinois.

Les spécialistes de l'histoire des religions, et plus spécifiquement de l'histoire du bouddhisme, offrent trop souvent du geyi une interprétation technicienne, étroitement philologique et normative. Ils y voient les premiers balbutiements de la traduction bouddhiste, au seuil d'une longue série évolutive, et cette méthode, ouverte à tous les contresens, n'aurait fleuri qu'à l'époque où les Chinois ne connaissaient pas la version sanskrite des sâtras, et où les introducteurs (parthes, sogdiens ou scythes) avaient du chinois une connaissance si approximative qu'elle favorisait tous les glissements de sens. Contre cette interprétation trop positiviste, il convient de souligner que la mutation que représente le passage à la nouvelle école de traduction (Dao'an et Kumarajiva) ne saurait être comprise uniquement en termes de progrès. L'abandon du geyi résulte moins d'une amélioration graduelle que d'un saut systémique accompagné par le choix de nouveaux critères.

bouddhistes. C'est pourquoi Ya, avec Kang Falang et quelques autres, prit les catégories contenues dans les écritures, les compara, les apparia avec les livres extérieurs, donnant ainsi des exemples pour favoriser la compréhension; il appela cette méthode «geyi» (cité par Tang Yung Tung, 1940, p. 276).

19. Xuan xue (mot à mot «étude obscure»): forme de syncrétisme néo-daoïste qui marqua les élites chinoises autour du III siècle de notre ère. 
Plusieurs indices viennent appuyer cette lecture. Tout d'abord, le geyi est clairement défini dans le Gaoseng zhuan comme une stratégie pédagogique et non comme un pari herméneutique. De plus, le phénomène geyi montre à merveille qu'un système de pensée étranger doit d'abord se révéler assimilable (et désirable) avant de se prêter à une analyse philologique. Enfin et surtout, nous croyons que le geyi reflète une tendance syncrétique lisible, en filigrane, dans toute l'histoire de la pensée chinoise (et a fortiori japonaise). Même après 383, lorsque la fondation du Xiaoyao Yuan à Chang'an aura sonné le glas de l'«ancienne traduction", la tendance assimilatrice perdurera, dans les zones laissées libres par le positivisme philologique. Sous les Wei septentrionaux, par exemple, Tan Qing rédigera un faux sûtra (le sûtra de Trapusa et Ballika) dans lequel les cinq vertus cardinales de la morale confucéenne seront rapprochées des cinq préceptes fondamentaux du bouddhisme.

Plus tard, lorsque le christianisme pénétrera en Chine, $c^{\prime}$ est le bouddhisme qui prêtera ses catégories a la traduction des mots-clés de la nouvelle religion: Dieu sera représenté par l'idéogramme du bouddha (fo), l'expression «Sauveur» par le binôme "seigneur des mondes" (shezun = Lokajyestha), le concept de rédemption par le composé «traversée universelle» (pudu).

Au-delà des spécificités du transfert notionnel, l'on sait que les Chinois se sont intéressés aux sectes mahayanistes qui se rapprochaient le plus des systèmes de pensée vernaculaires. Le succès du madhyamika ${ }^{20} s^{\prime}$ explique en grande partie par le fait qu'il permet de prolonger la réflexion sur la «médiété» (zhong) issue de l'horizon intellectuel confucéen. Quant au Chan méridional, il réintégrera des éléments daoïstes avant de se marier avec l'instantanéisme de Wang Yang Ming.

A un niveau plus général encore, le syncrétisme s'exprimera dans la thèse qui postule une complémentarité des

20. Madhyamika: théorie de la Voie Moyenne dans le bouddhisme du Grand Véhicule (tendance représentée par Nagarjûna). 
trois religions (sanjiao izhi lun/sankyoichiron) ${ }^{21}$ : à ce stade, le processus de digestion sera achevé, ou plutôt, l'antériorité de son «être déjà là» sera confirmée.

\section{Citation et clinamen conceptuel}

L'on ne soulignera jamais assez l'ambiguïté du geste de citation par lequel une culture dite périphérique se constitue dans son rapport au modèle central. Si le premier effet de la citation est de conférer une certaine légitimité à un énoncé en le référant à une tradition déjà reconnue, elle implique souvent un travail de sape et de dérivation qui, pour n'être pas toujours conscient, n'en est pas moins corrosif.

L'un des exemples les plus caractéristiques de ce phénomène se donne à lire dans le Nihonshoki. Annale japonaise presque entièrement sinisée, dans la langue d'une part (le texte est rédigé en pur kambun, à la différence du Kojiki), dans la conceptualisation d'autre part (la cosmogonie est codée en termes daö̈stes), le Nihonshoki ne se contente pas d'imiter l'écriture et les principes de l'historiographie chinoise, il va jusqu'à citer des passages entiers empruntés aux classiques chinois. Or, là même où l'influence continentale est la plus forte (la cosmogonie), le texte japonais déforme substantiellement la logique des ouvrages daoïstes de référence. La toile de fond et la panoplie conceptuelles semblent rigoureusement identiques: cosmogonies chinoise et japonaise nous parlent d'une gradation des origines, $d^{\prime}$ 'une opposition entre les corps lourds et les corps subtils, d'une séparation du Yin et du Yang, et accordent aux métaphores germinatives une place centrale. Il faut une comparaison plus fine du Lie zi, du Huai Nan zi, du San $W u$ Li Ji et du Nihonshoki pour percevoir le travail de dérivation.

21. Pour les Chinois, il s'agit du confucianisme, du bouddhisme et du taoissme. Pour les Japonais, la même expression désigne la complémentarité entre confucianisme, bouddhisme et shintoïsme. 
Lie zi (Tian Rui 1,2): «Ce qui était pur et léger monta et devint le ciel; ce qui était grossier et lourd descendit et devint la terre; les souffles médians formèrent l'homme. Grâce aux semences recelées par le ciel et la terre, les dix mille êtres apparurent par transformation.»

Huai Nan zi (éd. SSKT, 54, p. 130): «[...] le souffle originel était quelque chose de délimité. Ses éléments clairs et purs $\mathrm{s}$ 'élevant légèrement formèrent le ciel; ses éléments lourds et grossiers se coagulant formèrent la terre. Comme il est plus aisé aux éléments clairs et purs de se concentrer qu'aux éléments lourds et grossiers de se coaguler, le ciel se forma avant que la terre ne se stabilisât $[. . .]^{22}$.»

San Wu Li Ji (in Saigo, KCS, 1976, I, p. 83): «[...] ensuite fut produit le souffle originel. Le souffle originel germa alors pour la première fois (et donna naissance au ciel et à la terre).»

Nihonshoki (NKBT, N.I, pp. 76-77: Na): «Jadis, à l'époque où ni le ciel et la terre ni le yin et le yang n'étaient encore séparés, la matière chaotique ressemblait à un œuf: elle était confuse et contenait des germes. Les éléments purs et clairs s'élevèrent légèrement et formèrent le ciel. Les éléments lourds et grossiers se déposèrent et formèrent la terre."

Affinons le contraste entre la version du Nihonshoki et celle du San Wu Li Ji. Alors que la cosmogonie chinoise propose une vision transformative de la genèse (ming xing shi ya meng hong zi meng), le texte japonais présente la materia prima comme une sorte de khôra qui contient déjà des germes:

InisiFe ni amëtuti imada wakarezu mewo wakarezarisiki töki marökaretaru kötö töri nö ko nö götöku site Fönökani site kizasi wo Fukumëri.

22. La traduction des textes chinois est celle de M. Kaltenmark (in «La Naissance du Monde», Seuil, 1959, pp. 464-465). 
La citation traduit ainsi la référence chinoise dans la logique germinative du shintoïsme primitif. Les métaphores germinatives jouent en effet un rôle essentiel dans la Weltanschauung japonaise archaïque, et cela, au-delà de l'épisode de la genèse. Sous la forme de mönözane, l'idée de germe apparaît dans l'épisode de l'ukëFi, appliquée aux attributs (joyaux et épée) mâchés par les dieux lors de leur parthénogénèse rituelle. Le mönözane condense les propriétés de la cause matérielle et de ce que saint Bonaventure appellerait la cause séminale. Le terme lui-même, qui se décompose en «mönö nö tane» (germe/pépin de la chose), se voit défini comme principe de génération dans le IKGJ (1286) ou comme matériau originel dans le JKDJ ${ }^{23}$. Quoi qu'il en soit, la notion elle-même implique une analogie entre l'origine et l'«originé».

Les textes archaïques connaissent également la lecture mönösirö. Sous le règne de Sujin, par exemple, la terre du mont sacré Kaguyama est qualifiée de "Wa nö kuni nö mönösirö» (germe du pays de $\mathrm{Wa}$ ). C'est avec cette terre consacrée que l'on confectionne les plats rituels destinés aux kami. La valeur dynamique de la générativité fonctionne ici à plein, car le mönösirö est à la fois matière et énergie. La terre du mont Kagu était censée posséder des pouvoirs mystérieux, et on lui attribuait une valeur apotropaïtique.

Alors que la notion de mönözane restait chargée de connotations matérielles, le terme voisin de «kizasi» (lecture japonaise du caractère "ya» présent dans les textes daö̈sants cités plus haut) est plus abstraite et plus complexe. Utilisé par les lettrés pour rendre synthétiquement les concepts daoïstes de «ki» (qi) et de «kata» (xing), kizasi possède en fait une signification indépendante et vernaculaire. Il est composé de «ki» (germe, pointe) et du nom verbal «sasi» (pointer, viser, tendre vers, etc.) qui décrit le processus de génération ou la manifestation d'une énergie.

23. IKGJ: Iwanami Kogojiten; JKDJ: Jidaibetsu Kokugo Daijiten. 
Saisissons maintenant l'arrière-plan philosophique de ces différences entre Weltanschauung chinoise et japonaise. Alors que le discours cosmogonique daoïste débouche sur une «ontogonie» universaliste, la notion de "wan wu» (les dix mille êtres) ne joue aucun rôle dans la genèse japonaise. La génération du monde selon le Lie zi et le Huai Nan zi est tout entière orientée vers l'anthropogénèse, qui la couronne et en constitue la raison d'être et la cause finale. Les textes japonais abandonnent cette articulation de la cosmogonie sur l'anthropologie: ils introduisent une coupure radicale entre les réflexions sinisées sur les métamorphoses de la physis d'une part, et le mythe d'origine du pays de l'autre. Tout se passe comme si la logique philosophique et la mytho-logique ne se mêlaient pas, restaient simplement juxtaposées sans se féconder réciproquement. UFidini nö kamï et SuFidini nö kamï peuvent bien représenter la séparation du yin et du yang, ils sont dénués de la moindre épaisseur dramatique et conceptuelle. Inversement, Izanaki et Izanami ${ }^{24}$ sont des personnes concrètes et non des forces cosmiques: ils n'ont avec le yin et le yang que le point commun dérisoire d'être l'un mâle et l'autre femelle. La série généalogique daoïste se trouve ainsi, au terme du transfert, amputée à ses deux extrémités: en amont, les textes japonais abandonnent la quête de l'originarité de l'origine et, en aval, ils empêchent la cosmogonie de se déployer jusqu'à l'homme.

\section{La contextualisation neutralisante}

La citation dévoyée n'est pas la seule stratégie utilisée par l'emprunteur. Il arrive souvent qu'une culture périphérique absorbe «fidèlement» une notion, un système de pensée ou une institution, mais l'intègre dans un ensemble qui en neutralise la charge négative. Ce travail de désamorçage peut n'être que

24. Izanaki et Izanami sont les Welteltern de la cosmogonie japonaise. Ils enfantent les îles et les dieux de la nature. Après leur divorce, qui fonctionne comme un mythe d'origine de la mort, Izanaki donne naissance seul aux ancêtres de la famille impériale tandis que son épouse est affectée au royaume souterrain. 
symbolique, se réduisant parfois à la simple adjonction d'un signe de «vernacularisation», mais le mécanisme reste identique.

Dans le domaine institutionnel par exemple, lorsque le Japon protohistorique s'efforce de mettre sur pied une structure étatique à la chinoise, dans la foulée des réformes de Taika ${ }^{25}$, il importe un appareil politique et bureaucratique que les historiens appellent l'Etat des Codes (Ritsuryô Kokka) ${ }^{26}$. Ce système centralisé est entièrement continental dans ses principes et sa morphologie. Et pourtant, en intégrant dans cet appareil exogène une institution radicalement anti-chinoise, le Bureau des Affaires Religieuses (Jingikan), les Japonais soulignent leur volonté de contrecarrer le processus d'acculturation et de renforcer le travail de fiction identitaire: la fonction principale du Jingikan était de superviser les grandes cérémonies du culte vernaculaire, et de contrôler le réseau des sanctuaires shintô.

C'est dans le même contexte idéologique que les souverains, de Temmu à Gemmei, commanditeront la rédaction des Annales. Malgré la force d'attraction du modèle continental, qui se donne à lire dans l'utilisation du calendrier chinois pour remplacer la temporalité autoréférentielle du Kojiki ${ }^{27}$, les textes japonais renient complètement les principes de l'historiographie chinoise. Alors que la Chine tourne le dos a toute pensée de l'origine (daoïsme excepté, mais la vision daoïste $n^{\prime}$ intervient pas

25. L'expression «Réformes de Taika» désigne une série de mesures centralisatrices prises entre 645 et 647 sur l'initiative de Naka no Ohoe (le futur Tenchi).

26. Ritsuryô désigne en japonais le double code, pénal (ritsu) et administratif (ryô) inspiré des Tang et fixé en 702 sous le nom de code Taihô. Les historiens utilisent la métonymie "Ritsuryôkokka» pour qualifier l'État centralisé subsumé par ces codes, et l'appareil bureaucratique constitué par le Dajokan, le Jingikan, les huit Ministères et les Bureaux.

27. Alors que le Nihonshoki utilise le calendrier chinois, la temporalité du Kojiki ne connaît pour toute mesure que la durée de vie des empereurs. 
dans l'historiographie) pour penser le temps comme pur procès sans début ni fin, et pour enfermer son imaginaire dans des encyclopédies du bizarre, le Japon choisit de redonner vie à ses mythes et de les placer en prologue à la généalogie dynastique, fondant sa spécificité nationale sur la notion d'Âge des dieux (kami no yo). Une analyse détaillée des deux premiers livres du Nihonshoki révèle un ordre du discours plus proche de la pensée sauvage que des règles du Shi Tong ${ }^{28}$. Le premier livre est embrassé par deux genèses, une macrocosmogonie (premières générations et kuni-umi $)^{29}$ et une microcosmogonie (kunizukuri ${ }^{30} \mathrm{~d}^{\prime}$ OFokuninusi) qui flanquent le drame central de l'iFayato ${ }^{31}$, drame qui se décompose à son tour en deux scènes antithétiques (la mort et la renaissance d'Amaterasu). Le second livre révèle la même symétrie puisqu'il est encadré par deux cessions, la cession du pays par oFokuninusi d'une part, la cession de l'aînesse par Foderi d'autre part, épisodes entre lesquels est sertie la double catabase (descente ratée d'OsiFomimi et descente réussie de Ninigi) ${ }^{32}$.

Une lecture plus fine confirme que la mytho-logique vernaculaire sape la chrono-logique des Annalistes sinisés. Le livre I du Nihonshoki, qui est centré sur la dimension céleste, est placé sous le signe de l'inceste; il raconte les vicissitudes de l'instauration du cosmos. Le livre II, dont les épisodes se déroulent sur la terre, introduit le thème de l'exogamie. Il met en

28. Shi Tong: ouvrage de la dynastie Tang, qui présente les grands principes de l'historiographie chinoise.

29. Kuni-umi: enfantement des pays (îles du Japon).

30. Kunizukuri: aménagement du pays.

31. iFayato: mythe racontant la retraite de la déesse du soleil dans la grotte céleste (et sa mort symbolique) ainsi que les rites de rappel qui lui font suite.

32. Cette descente sur le mont Takachiho accomplie par le petit-fils de la déesse du soleil est à la fois une prise de possession du pays, un rite agraire et la version mythologique de la cérémonie impériale du Daijôsai. 
scène la pacification et la prise de possession du monde. Les épisodes symétriques qui occupent nos livres reposent tous sur une tentative matrimoniale ratée, s'accompagnent tous $d^{\prime} u n$ voyage dans l'au-delà, et s'achèvent tous sur une métamorphose du protagoniste. Izanaki revient à la vie et accomplit une parthénogenèse qui corrige le kamï-umi. Amaterasu ressuscite, tandis qu'à la fin du cyle Fowori obtient la souveraineté en même temps que le droit d'aînesse...

Ainsi les compilateurs habillaient-ils de mots chinois une histoire sacrée entièrement traversée de notions shintoïstes.

\section{La réappropriation par inversion}

Lorsque la culture emprunteuse a suffisamment maîtrisé le système notionnel exogène, elle lui fait subir le sort qu'elle lui réservait dès le moment du transfert: l'armature globale du système est préservée, mais elle se trouve inversée, ou affectée d'une valorisation contraire.

L'évolution du syncrétisme shinto-bouddhiste au Japon illustre parfaitement ce phénomène. Dans un premier temps, les dieux du shintô se voient assigner le rôle de protecteurs de la loi bouddhique et des grands temples. Mais, dès l'époque de Nara, parallèlement à la conception du kami protecteur, un premier glissement vers une forme de complémentarité hiérarchique commence à se manifester. Le clergé bouddhique adopte à l'égard du shintoïsme l'attitude que le bouddhisme continental avait prise pour assimiler les dieux indiens. On reconnaît leur statut d'entités surnaturelles, mais on les présente comme des créatures qui n'ont pas encore atteint la libération. Leur karma les condamne momentanément à une existence de divinités inférieures. Ainsi se développe un culte spécifique destiné à accélérer la libération des kami: c'est dans ce but que l'on érige des Jingûji et des Shinganji dans les sanctuaires. Une conception voisine établit une équivalence entre les bodhisattva et les kami: dieux de compassion, ils refusent momentanément la libération pour mieux travailler à sauver les hommes. 
Mais c'est à la fin du VIII e siècle que l'assimilation s'accélère et affecte la théonymie, comme le montre l'appellation de «Hachiman bosatsu» réservée au kami Usa Hachiman. Au milieu de la période Heian apparaît pour la première fois l'idée que les kami ne sont que la manifestation (gongen, kegen) ou la trace (suijaku) des bouddhas qui, eux, se voient qualifier de «honji» (source, prototype), mais l'équivalence postulée entre kami et bouddha reste générique. Ce n'est qu'à la fin de la période Heian (XII ${ }^{e}$ siècle) que s'élabore un véritable système de correspondances nominales entre les kami et les bouddha. La période Kamakura marque l'apogée et l'ultime perfectionnement de la théorie du «honji-suijaku», qui est représentée par deux formes de shintoïsme syncrétique, le Ryôbu Shintô, proche de la secte Shingon à laquelle il emprunte les catégories de nouménal et de phénoménal (ryôbu), et le San'ô Shintô qui évolue dans $l^{\prime}$ orbe du Tendaï. Le cercle sera bouclé quand, au $X V^{e}$ siècle, Yoshida Kanetomo (1435-1511) tentera de restaurer le shintoïsme dans son intégrité en gardant l'armature globale du honji-suijaku. Le chantre du renouveau shintoïste n'aura plus qu'à renverser le rapport hiérarchique des termes du Ryôbu afin de présenter les bouddha comme les "traces manifestes» des kami. Dans un effort désespéré pour retrouver sa pureté première, le shintô laissait le visible aux bouddha et tentait de construire une théologie en monopolisant l'invisible.

\section{L'assimilation antagoniste}

La forme la plus complexe de transfert notionnel, celle qui fait jouer à plein la logique de la déconstruction, est, dans l'horizon qui nous intéresse, la transformation progressive du néoconfucianisme durant les deux premiers siecles de la période Edo. Lors même qu'ils croient rester fidèles à l'héritage philosophique de $\mathrm{Zhu} \mathrm{Xi}^{33}$, les penseurs philologues $\mathrm{d}^{\prime} \mathrm{Edo}$ se livrent à un travail de démantèlement qui fait le lit de la réaction des Études Nationales, travail de démantèlement dont les grands

33. Zhu Xi (1130-1200): philosophe de la dynastie Song. On lui doit une réélaboration métaphysique du confucianisme en une synthèse qui utilise certaines notions bouddhistes et taoïstes. 
traits sont: rejet des principes universels et de la dimension métaphysique, désarticulation du politique et de l'éthique, critique de la morale de la maîtrise et développement d'une herméneutique de la tautégorie.

Ito Jinsai, en critiquant la dimension principielle du concept central du néo-confucianisme, le «li», en dévalorisant les "concepts statiques» (shiji) comme la raison, le vide, etc., qu'il propose de remplacer par des concepts dynamiques (katsuji) sur le modèle de «michi», s'attaque à l'édifice notionnel du grand penseur des Song. Lorsque Ogyû Sôraï, radicalisant le message légiste de sa théorie politique, affirme que les souverains n'ont d'autre règle que la "voie des rois», c'est-à-dire la gestion du Tout, et ne sont donc pas soumis au principe de la vertu (de/toku) qui ne s'applique qu'aux dominés, il instaure une coupure radicale entre le politique et l'éthique. Lorsque Ito Jinsai s'attaque à la thèse mencienne des «si tuan ${ }^{34}$ », réhabilite le désir en contestant l'opposition "tian li/ren yu» (raison céleste/désir humain), et dénonce le travail du «zheng $x^{35}{ }^{35}$ (orthopsychie) ainsi que l'idéal de l'ataraxie (mihatsu), il rejette globalement la morale de la maîtrise, qui est une des clefs de voâte du système néo-confucéen. Lorsque les philologues japonais condamnent les commentaires et remontent, avec le Kogigaku, puis le $\mathrm{Kobunjigaku}^{36}$, à un corpus de plus en plus ancien, de plus en

34. Si tuan (4 extrémités): ce sont les dispositions innées qui permettent l'éclosion des quatre vertus cardinales. La sympathie humaine naît de la compassion; l'équité provient de la sensibilité à la honte; le sens du rituel découle de la modestie; le discernement $\mathrm{du}$ bien et du mal est le commencement de la sagesse.

35. Zheng xin: correction, ou rectification du cœur. Cette conception implique une morale de la maîtrise, un travail de soi sur soi pour atteindre la vertu. Déjà présente dans le confucianisme ancien ("rechercher la sincérité par la rectification du coeur»), cette conception connaîtra surtout un grand succès pendant la dynastie Song.

36. Deux tendances importantes dans la philologie japonaise (d'Ito Jinsai à Ogyu Soraï). 
plus vierge d'interprétations, ils inaugurent une tendance herménophobe qui prépare la lecture tautégorique que Motoori fera plus tard des textes vernaculaires (Kojikiden) ${ }^{37}$.

Ainsi, l'édifice de la métaphysique de Zhu Xi n'aura pas été abattu de l'extérieur par la réaction nationaliste des Kokugakusha: il s'est trouvé démantelé, déconstruit de l'intérieur par les penseurs mêmes qui entendaient l'appliquer au Japon.

\section{Conditions philosophiques}

Bien que les phénomènes de réinterprétation que nous venons de décrire s'inscrivent dans une morphologie universelle des procédés d'emprunt, ils semblent favorisés par quelques paramètres philosophiques et culturels spécifiquement asiatiques.

La tendance à l'assimilation englobante trouve un adjuvant indéniable dans la vocation universelle du bouddhisme et dans la souplesse des doctrines qui le constituent. Car si le bouddhisme, au fil de sa dérive vers l'est, s'est prêté à toutes les réinterprétations vernaculaires, son contenu doctrinal et théorique le situe aux antipodes d'une religion «ethnique» comme le shintôisme. L'abstraction de la théorie des «skandha» (éléments constitutifs de tous les êtres), la généralité éthique de la conception du bodhisattva et la nature du mécanisme de rétribution karmique font du Grand Véhicule un englobant universel que n'affectent en rien les traductions ou les insertions dans de nouveaux contextes. L'on comprend que dans un tel horizon intellectuel, malgré la conscience des spécificités linguistiques ou philosophiques exposées dans le «wu bu fan»

37. Le Kojikiden est un commentaire monumental du Kojiki, écrit par Motoori Norinaga (1730-1801), le grand penseur du Kokugaku. On y trouve une lecture philologique extrêmement fine du texte et une approche tautégorique du mythe qui rompt avec les lectures allégorisantes des savants confucéens. 
(cinq cas d'intraduisibilité) $)^{38}$ de Xuan Zhang, la traduction n'ait pas été pensée comme une transformation radicale: la majorité des moines traducteurs sont intimement persuadés de l'existence d'un métacode universel qui transcenderait ces discours particuliers que sont les sectes ou les langues. Dans ses «Biographies des moines éminents», Zanning ( $X^{e}$ siècle) définit ainsi l'acte de traduction:

Traduire signifie changer, c'est-à-dire changer ce que vous n'avez pas contre ce que vous avez. C'est comme dans le cas de l'orange trifoliée et de l'orange. Transplantée dans un terrain différent, l'orange se transforme en orange trifoliée. Malgré cette différence d'appellation, il n'y a pas de différence de parfum, de tronc, de feuilles [...]. («Biographies des moines éminents", DZ] Livre 50, pp. 723-724; cité par Yan Yang, 1992, pp. 69-70)

Ailleurs, il va même jusqu'à comparer la traduction à l'envers d'un brocard: «les motifs des deux côtés sont identiques, bien qu'ils soient différents dans leur direction».

Un autre paramètre explique l'innocence du jeu dérivatif qui accompagne le transfert: il s'agit du régime sémiotique de la sérialisation. Tout horizon culturel traditionnel a tendance à se reproduire par autophagie, réutilisant sans cesse les mêmes formes qu'il soumet à des combinaisons nouvelles. Dans la culture japonaise, cette propension est poussée à l'extrême, et on peut en déceler la trace dès les premiers textes écrits de la période de Nara, le Kojiki et le Nihonshoki. Utilisant un matériau déja offert par la tradition ( $\mathrm{V}^{\mathrm{e}}, \mathrm{VI}^{e}$ et $\mathrm{VII}^{\mathrm{e}}$ siècles), les compilateurs le recontextualisent pour lui octroyer une signification tout autre. Ainsi, les généalogies du Teiki vont fonctionner comme des scansions structurelles dans la rythmique

38. Les cinq cas d'intraduisibilité sont les suivants: 1) la notion est un secret qu'il ne faut pas dévoiler; 2) la polysémie du terme sanskrit le rend intraduisible; 3 ) le terme désigne un référent qui n'existe pas en Chine; 4) la tradition a imposé l'emploi du terme sanskrit; 5) le terme sanskrit est conservé pour éviter les connotations négatives de son correspondant chinois. 
narrative de la mythologie savante, les incantations rituelles vont se transformer en poèmes lyriques ( $d$ 'une Annale à l'autre, d'ailleurs, le même poème se verra intégré dans des contextes et des systèmes de sens différents) et les citations chinoises vont fonctionner dans une logique de la légitimation.

Cette disponibilité sémiotique de l'élément qui n'a de valeur que virtuelle, et qui appelle toujours une mise en série, caractérise également les pratiques poétiques classiques (des anthologies de Heian à l'esthétique du hokku en passant par le renga) et explique sans doute la facilité avec laquelle, même dans les domaines religieux ou philosophique, les auteurs japonais font subir maintes variations aux textes-thèmes que leur offrent les traditions locales ou étrangères.

C'est enfin et surtout le phénoménalisme fondamental de la pensée japonaise (et chinoise) qui constitue l'horizon de possibilité des pratiques dérivationnelles sur lesquelles nous nous sommes arrêté. Philosophies de l'immanence, tous les systèmes de pensée qui se sont développés au Japon n'ont jamais problématisé la question de la vérité, laissant cette case vide à la notion autoréférentielle de sincérité (makoto/cheng). Les mouvements antithéoriques et antiphilosophiques appuient leur critique des systèmes continentaux sur l'éthique de la sincérité qui débouche sur une pragmatique instantanéiste. C'est pour cela que la forme d'osmose la plus fréquente dans la pensée instantanéiste continentale ou japonaise est celle qui unit Wang Yang Ming au Zen.

Dans un tel horizon philosophique, l'acte de compréhension ne passe pas par une réflexion sur l'authenticité ou, quand il le fait, le dépasse pour fonder le rapport au texte sur la notion dynamique d'opérativité. Intelligere est facere auraient pu dire les philologues japonais, qui se rapprochent de la pensée de Vico dans la mesure où ils définissent implicitement la compréhension comme la possibilité de produire de nouvelles variantes à partir d'un thème donné par la tradition. 


\section{Conclusion}

Bien qu'ils proviennent d'une aire culturelle spécifique, les quelques cas que nous venons d'analyser nous autorisent à contester la métaphore organique sous-jacente à nombre de théories de l'emprunt. Le modèle trop souvent appliqué aux cultures périphériques postule en effet que tout organisme emprunteur passe par une étape d'imitation servile, de sélection et enfin d'assimilation avant de produire un nouveau système et d'atteindre ainsi sa maturité. L'exemple de la Chine et du Japon nous porte à croire au contraire qu'il n'y a pas d'enfance des systèmes, car l'emprunt est déjà prédéterminé par des structures de représentation et des stratégies d'utilisation complexes, qui rendent invraisemblable l'hypothèse d'un stade mimétique.

L'Extrême-Asie nous enseigne aussi qu'il n'y a pas d'erreur dans les phénomènes de tranfert culturel. Le solécisme ne saurait exister puisque les systèmes culturels, qui prouvent leur "vérité propre» par leur dynamisme, ressemblent plus à des systèmes cognitifs qu'à des systèmes sémiotiques.

Et enfin, le destin du bouddhisme et du confucianisme, la malléabilité extraordinaire de leurs variantes, suffisent à montrer la créativité de ces cultures encore trop souvent présentées en Occident comme des cultures de l'imitation. La copie n'existe pas: tout emprunt est transformation. Le geste du transfert implique non seulement une saisie analytique des premiers sens de la notion, mais encore l'intelligence projective qui consiste à lui assigner une nouvelle fin dans son système d'accueil.

Université de Montréal 


\section{Références}

ARAKI Kengo (1976). Bukkyô to Jûkyô: Chûgoku shisô o keiseisuru mono (Bouddhisme et Confucianisme: les éléments de la pensée chinoise). Kyoto, Heirakuji shoten.

CHEN, K. (1960). Some problems in the translation of the Buddhist Canon. Ch'ing hua hsüeh pao, Peiping, Taipei, vol. II (1), pp. 84103.

De BARY, W.F. (1975). The Unfolding of Neo-Confucianism. New York, Columbia University Press.

DEMIÉVILLE, Paul (1956; 1973). «La Pénétration du bouddhisme dans la tradition philosophique chinoise", in Demiéville, P., Choix d'études sinologiques. Leiden, Brill.

DUMOULIN, Heinrich, S.J. (1988; 1990). Zen Buddhism: a History. New York, Mac Millan (2 vol.).

FAURE, Bernard (1987a). «Space and place in Chinese religious traditions", History of Religions XXVI (4), pp. 337-356.

selon le Shôbôgenzô. Paris, Le Mail.

(1987b). La Vision immédiate: nature, éveil et tradition

(1989). Le Bouddhisme Ch'an en mal d'histoire: genèse d'une tradition religieuse dans la Chine des T'ang. Paris, E.F.E.O.

Jidaibetsu kokugo daijiten. Jôdaihen (1967). Tôkyô, Sanseidô.

Kodai kayô-shû (1957). Nihon kotenbungaku taikei 3. Tôkyô, Iwanami shoten.

Kojiki (1982). Nihon shisô taikei 1. Tôkyô, Iwanami shoten.

Kojiki-Norito (1958). Nihon kotenbungaku taikei 1. Tôkyô, Iwanami shoten. 
KÔNOSHI, Takamitsu (1983). Kojiki no tassei. Tôkyô, Tôkyô daigaku shuppankai.

Kôza Nihon no shinwa (1976-1978). Tôkyô, Yûseidô (12 vol.).

LAFLEUR, W.M., dir. (1985). Dôgen studies. Honolulu, University of Hawaii Press.

LAMOTTE, Étienne (1958). Histoire du bouddhisme indien: des origines à l'ère Saka. Louvain, Institut orientaliste.

MacWILLIAMS, Mark W. (1990). «Kannon Engi. The reijô and the concept of kechien as strategies of indigenization in buddhist sacred narrative», Transactions of the Asiatic Society of Japan, vol. V, pp. 53-70.

MATSUMURA, Takeo (1954-1958). Nihonshinwa no kenkyû. Tôkyô, Baifûkan (4 vol.).

MISHINA, Akihide (1970). Nihonshinwaron. Tôkyô, Heibonsha.

Motoori Norinaga Zenshû (1968). Tôkyô, Chikuma shôbô.

MUNRO, David J. (1985). Individualism and Holism: Studies in Confucian and Taoist Values. Ann Arbor, Center for Chinese Studies, University of Michigan.

NAKANISHI, Susumu (1985). Kojiki o yomu. Tôkyô, Kadokawa shoten (4 vol.).

Nihonshoki (1967). Nihon kotenbungaku taikei 67-68. TOkyô, Iwanami shoten.

ONO, Susumu, dir. (1974). Kogojiten. Tôkyô, Iwanami shoten.

SAIGO, Nobutsuna (1976). Kojiki chûshaku. Tôkyô, Heibonsha.

TANG, Yung-Tung (1940). «On «Ko-Yi», the Earliest Method by which Indian Buddhism and Chinese Thought were synthetized", 
In Honour of Radhakrishnan, Comparative Studies in Philosophy, pp. 276-286.

TSUNODA, de Bary, Keene, dir. (1958). Sources of Japanese tradition. New York, Columbia University Press.

YANG, Yan (1992). A Brief History of Chinese Translation Theory. Ph. D. Dissertation. The University of Texas, U.M.I.

ZÜRCHER, Erik (1959). The Buddhist conquest of China: the spread and adaptation of Buddhism in early medieval China. Leiden, Brill (2 vol.). Pao 66, 1-3, pp. 84-147.

(1980). «Buddhist Influence on Early Taoism», T'oung

RÉSUMÉ: La trahison créatrice: anatomie du transfert notionnel dans les cultures asiatiques - Nous proposons une lecture constructiviste du phénomène de transfert conceptuel dans les traditions philosophiques et religieuses de la Chine et du Japon. Par transfert, nous entendons aussi bien l'emprunt d'une notion isolée et son insertion dans un nouveau système culturel que la translation d'un ensemble catégoriel complexe. Nous nous intéressons surtout aux distorsions sémantiques qui se produisent lors du transfert (et à ces cas extrêmes que sont les transformations délibérées) pour illustrer l'hypothèse suivante: l'importation d'un concept est toujours un acte de création. Nous soulignons l'importance des structures intentionnelles qui marquent le geste de translation: prospection, pré-interprétation, stratégies d'utilisation, etc. Cette hypothèse nous permet d'étudier la traduction philosophique en faisant l'économie de la problématique de la vérité, et ensuite de contester l'étiquette de «cultures de la copie» trop souvent apposée aux traditions d'Extrême-Asie.

ABSTRACT: Creative betrayal: anatomy of notional transfer in Asian cultures - This essay is an attempt at a constructivist analysis of the "conceptual transfer" in the philosophical and religious traditions of China and Japan. The term "transfer" here implies the borrowing of an isolated notion and its insertion into a new cultural system as well as the 
translation of a whole complex of categories. We accord special interest to the different types of semantic distorsions which occur during the transfer (and especially to deliberate transformations) in order to substantiate our working hypothesis, namely, the import of a concept is more akin to an act of creation than to a mere reproduction. We underline the importance of the intentional structures which characterize the transfer: prospection, pre-interpretation, strategies of application, etc. This hypothesis enables us to analyze the phenomenon of philosophical (or religious) translation without relying on the concept of authenticity, and permits us to reevaluate the creative impulse of the cultures of China and Japan, that have too often been described as "copy cultures." 